\title{
Molecular Recognition and Interfacial Catalysis at Liquid-Liquid Interfaces
}

\author{
Alexander G. VolKov \\ Department of Civil and Environmental Engineering, $5732 \mathrm{~F}$ Boelter Hall, \\ University of California at Los Angeles, Los Angeles, CA 90095-1593, USA
}

\begin{abstract}
The interface between two immiscible liquids with immobilized metalloporphyrins, enzymes and submitochondrial particles can serve as the simplest model of biological membrane convenient for the investigation of redox reaction accompanied by spatial separation of charges. In this mini review, we have described the vectorial charge transfer and molecular recognition at liquid/liquid interfaces. The structure of liquid interfaces, donor-acceptor interactions in the electrical double layer, hydrogen and specific bounding between molecules play the dominant role in molecular recognition and interfacial catalysis.
\end{abstract}

Keywords Oil/water interface, Kelvin probe, vibrating condenser, molecular recognition, interfacial catalysis, cytochrome oxidase, porphyrins

1 Introduction

2 Kelvin probe as an non-invasive electroanalytical method

19 20

3 Submitochondrial particles: molecular recognition and interfacical catalysis

4 Enzyme complexes of the mitochondrial respiratory chain
5 Porphyrins as interfacial catalysts 22

6 Reduction of porphyrin at the octane/water interface controlled by the specific adsorption: molecular recognition of anions at the octane/water interface

7 Conclusion 26

\section{Introduction}

Vectorial charge transfer and a molecular recognition at the interface between two dielectric media are important stages in many bioelectrochemical processes such as those mediated by energy transducing membranes. Boundary membranes play a key role in the cells of all contemporary organisms, and simple models of membrane function are therefore of considerable interest. The interface of two immiscible liquids has been widely used for this purpose. For example, the fundamental processes of photosynthesis, ion pumping and electron transport have all been investigated in such interfacial systems. ${ }^{1-6}$ Studies have been made on molecular recognition during redox and hydrolysis reactions catalyzed by enzymes, photosynthetic pigments, metal complexes of porphyrins, bacteria, submitochondrial particles, as well as in systems with an extended surface - in microemulsions, vesicles and reversed micelles. Enzymes and pigments embedded in a hydrophilic-hydrophobic interface have properties similar to their functional state in a membrane. Molecular organization at liquid/liquid interfaces provides unique advantages for molecular recognition, which is the sci- ence of complementarity. ${ }^{1,5}$

From thermodynamic and kinetic principles the interface between two immiscible liquids can have catalytic properties for interfacial charge transfer reactions. It is possible to shift the redox potential scale in a desired direction by selecting appropriate solvents, thereby permitting reactions to occur that are highly unfavorable in a homogeneous phase. As it follows from thermodynamics, if the resolvation energies of substrates and products are very different, the interface between two immiscible liquids may act as a catalyst. The kinetic mechanism underlying the catalytic properties of the liquid/liquid interface and the molecular recognition was discussed first by Kharkats and Volkov. ${ }^{7,8}$

Redox reactions at the interface between immiscible liquids fall into two classes. The first class includes spontaneous processes that occur in the absence of external electromagnetic fields. This type of redox transformation has been investigated in bioenergetics, model membrane systems and at oil/water interfaces. Redox reactions in the second class occur at the interface between immiscible electrolytes when external electrical fields are applied to the interface, and under these conditions interfacial charge transfer reactions take place at controlled interfacial potentials. 
Electrochemical interfacial reactions are usually multistage processes that have the following steps: (i) diffusion of reactant to the interface; (ii) adsorption of reactants onto the interface; (iii) molecular recognition and chemical reaction at the interface; (iv) desorption of products from the interface; (v) diffusion of products from the interface.

\section{Kelvin probe as an non-invasive electroanalytical method}

All the methods of measuring Volta potentials between two phases $\alpha$ and $\beta$ use compensation. This means that a potential difference is applied between the phases $\alpha$ and $\beta$ which is equal in magnitude to the Volta potential but has an opposite sign. Changes in Volta potentials and Galvani potentials at liquid/liquid interfaces can be measured by the dynamic capacitor method, proposed by Kelvin and later developed by Zisman. This method is called also dynamic condenser, Kelvin probe, Kelvin bioprobe, vibrating electrode, or vibrating capacitor method.

A schematic diagram of the setup for measuring the Volta potential by the dynamic capacitor method is shown in Fig. 1. A generator of mechanical vibrations drives a gold electrode to vibrate at a distance of $0.2-1.0 \mathrm{~mm}$ from the surface of the liquid phase in question. To eliminate the effect of the diffusion potential, the aqueous solution is connected to a reversible reference electrode via a salt bridge filled with saturated $\mathrm{KCl}$ solution. The displacement current, which occurs due to the vibration of the gold electrode, is compensated by applying to the vibrating electrode a potential equal in magnitude to the measured Volta potential but having an opposite sign.

Electron and ion transfer reactions have been studied by using the vibrating plate method in the electrochemical circuit:

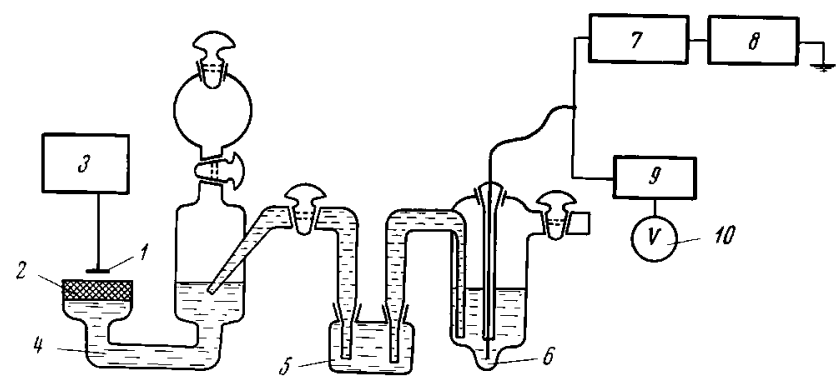

Fig. 1 Schematic diagram of the setup for measuring Volta potentials between two immiscible liquids. Notation: 1, vibrating gold electrode; 2, layer of oil; 3, generator; 4, aqueous solution; 5 , salt bridge; 6 , calomel electrode; 7 , amplifier; 8, oscillograph; 9, a compensating device. The compensating potential was measured by a voltmeter (10).

$$
\mathrm{Au} \mid \text { Air }\left|\begin{array}{l|l|l|}
\text { Octane } & \text { Water } \\
\text { Acceptor } & \text { Substrate } \\
\text { Catalyst } & \text { Tris-HCl }
\end{array}\right| \text { sat. } \mathrm{KCl} \mid \mathrm{Hg}_{2} \mathrm{Cl}_{2}, \mathrm{Hg}
$$

If a hydrophobic acceptor of electrons such as vitamin $\mathrm{K}_{3}$ is added to the octane and a donor of electrons to the aqueous phase, the Volta-potential measured in the chain (I) shifts in the negative direction due to the catalytic electron-exchange reaction at the oil/water interface:

$$
\operatorname{Red}_{\mathrm{aq}}+\mathrm{Ox}_{\mathrm{oil}} \stackrel{\text { Interfacial catalyst }}{\longrightarrow} \operatorname{Red}_{\mathrm{aq}}{ }^{+}+\mathrm{Ox}_{\mathrm{oil}}{ }^{-}
$$

The magnitude of the potential usually does not depend on how the redox reaction is initiated, that is by addition of substrates, catalysts or charge acceptors.

\section{Submitochondrial particles: molecular recognition and interfacial catalysis}

Experiments with submitochondrial particles (SMP) showed that in the course of the redox reactions at the octane/water interface, catalyzed by specific enzymes of SMP, the charges are transferred from the aqueous to the octane phase. ${ }^{9}$ The effects were detected by a shift of the Volta-potential, using the vibrate electrode method. In the presence of 1,4-naphtoquinone in octane, acting as an electron acceptor, the negative charges transfer from donors in aqueous phase to acceptors in an octane phase following the oxidation of $\mathrm{NADH}$, succinate and ascorbate. The generation of the electric potential difference is sensitive to the inhibitors of the respiratory chain, e.g. rotenone, antimycin and cyanide. ${ }^{9}$ In the presence of 2,4-dinitrophenol in octane, acting as a proton acceptor, the oxidation of NADH and succinate by ferricyanide, catalyzed by SMP in the presence of antimycin and cyanide correspondingly, was followed by a transfer of positive charges from aqueous phase to protonofore molecule in an octane phase. The positive charging of the octane phase, coupled with NADH oxidation, was found insensitive to rotenone, and that coupled with succinate oxidation, was completely inhibited by antimycin. The positive charging of the octane phase was also observed during the reverse transhydrogenase reaction, catalyzed by SMP at the interface. The effect was inhibited by palmitoyl-CoA. ${ }^{9}$

It seems likely that SMP in interacting with the interface undergo specific structural rearrangements, as a result of which functional contact of specific segments of the respiratory chain with the charge acceptors in the aqueous and octane phases is provided. In particular, it was found that measurement of the electrogenic function of the SMP at the interface becomes impossible when the salt incubation medium is replaced by 
sucrose. The salt effects probably provide the structural rearrangements of the SMP membrane necessary for the functional contact with the octane phase. The mitochondrial respiratory chain has specific segments of functional groups which provide the charge transfer from the aqueous to the lipid phase. The principal function of these enzyme systems as molecular generators of an electric field in coupling biomembranes is probably manifested in the catalysis of charge transfer across an interface.

\section{Enzyme complexes of the mitochondrial respiratory chain}

The function of the enzymes of the mitochondrial respiratory chain is to transform the energy of redox reactions into an proton gradient across the hydrophobic barrier of a coupling membrane. Isolated oligoenzyme complexes of the respiratory chain of mitochondria cytochrome $c$ oxidase, succinate-cytochrome $c$ reductase, and NADH-CoQ reductase are able to catalyze charge transfer of charges between water and octane, which can be followed by the change in the potential at the octane/water interface. ${ }^{10,11}$ A necessary condition for this measurement is the presence of the enzymes and substrates in the aqueous phase and a charge acceptor in the octane phase.

Cytochrome oxidase (EC 1.8.3.1) is the terminal electron acceptor of the mitochondrial respiratory chain. Its main function is to catalyze the reaction of dioxygen reduction to water using electrons from ferrocytochrome $c$ :

$$
4 \mathrm{H}^{+}+\mathrm{O}_{2}+4 \mathrm{e}^{-} \underset{\text { photosynthesis }}{\stackrel{\text { respiration }}{\rightleftharpoons}} 2 \mathrm{H}_{2} \mathrm{O}
$$

Aerobic oxidation of cytochrome $c$ is accomplished by cytochrome $c$ oxidase and represents the terminal step of the respiratory chain in mitochondria. Cytochrome $c$ oxidase can be considered as being the acceptor and donor of four electrons. Experiments with cytochrome $c$ oxidase in the octane/water system showed that, when an enzyme suspension is added to the octane/water system, some of the enzyme molecules adhere to the interface. As a result, when cytochrome $c$ is present, a negative Volta potential shift occurs upon addition of ascorbate. The dependence of $\Delta \phi$ on the cytochrome $c$ oxidase concentration is shown in Fig. 2. Effect depends on concentration of ascorbate (Fig. 3), cytochrome $c$ and $\mathrm{pH}$ (Fig. 4). The negative potential shift arising in the full system decreased to zero when inhibitors of cytochrome $c$ oxidase such as cyanide were added (Fig. 5).

The transfer of charges into octane catalyzed by the NADH-CoQ reductase complex requires special consideration. When a lipophilic electron acceptor is present in the octane, this system catalyzes the transfer of an electron into the octane by a route sensitive to

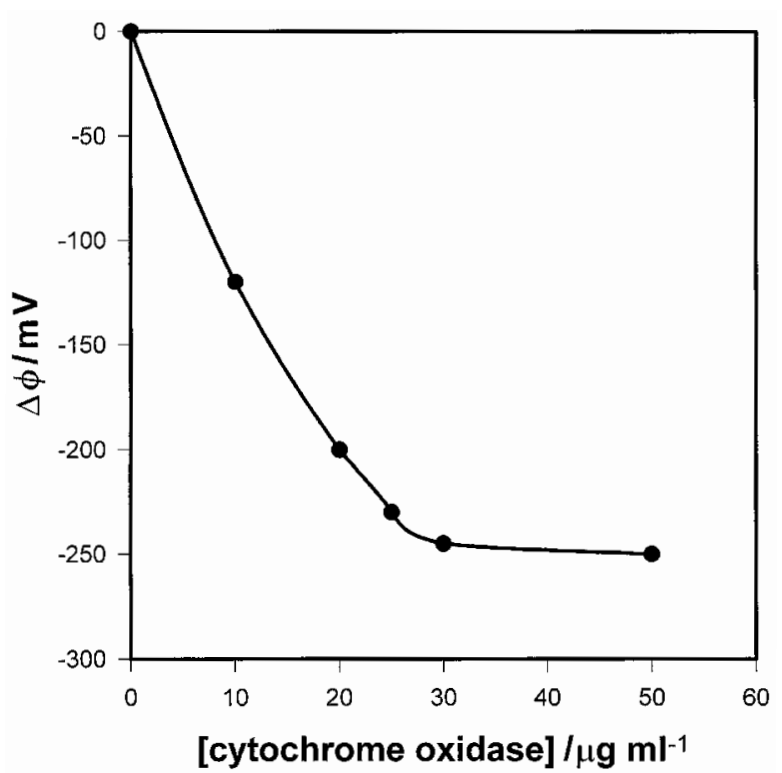

Fig. 2 Dependence of the Volta-potential at the octane/water interface on the concentration of cytochrome $c$ oxidase. Medium: $0.02 \mathrm{M}$ Tris- $\mathrm{HCl}$ ( $\mathrm{pH} 7.4$ ), $4 \mathrm{mM}$ ascorbate, 0.1 $\mathrm{mM}$ cytochrome $c$, and $10^{-4} \mathrm{M}$ vitamin $\mathrm{K}_{3}$.

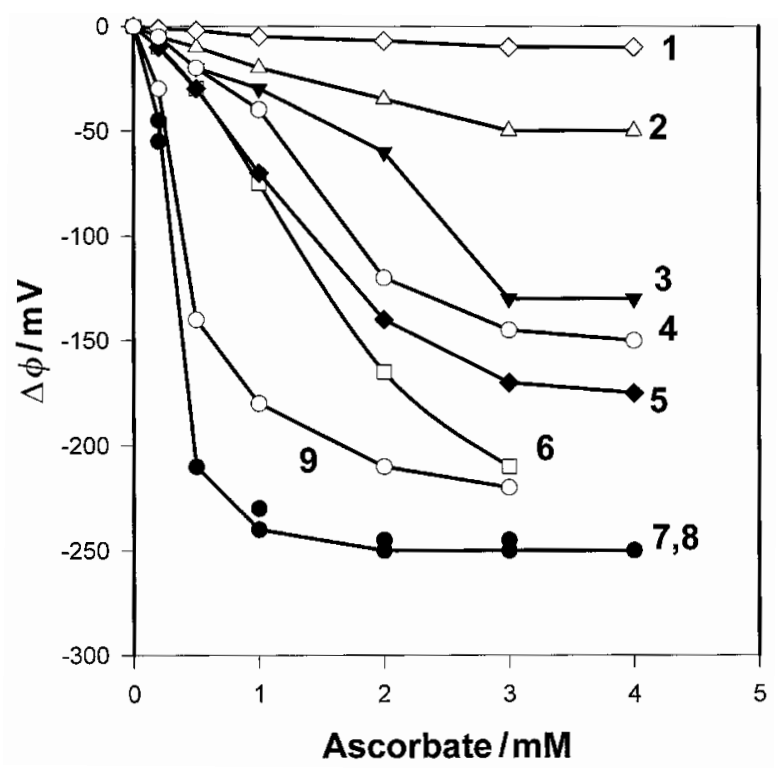

Fig. 3 Dependence of the Volta-potential at the octane/water interface on the concentration of ascorbate. Medium: $0.02 \mathrm{M}$ Tris-HCl, $40 \mathrm{mg} / \mathrm{ml}$ cytochrome oxidase, $0.1 \mathrm{mM}$ cytochrome $c$, and $10^{-4} \mathrm{M}$ vitamin $\mathrm{K}_{3}$. $\mathrm{pH}$ : (1) 6.0, (2) 6.4, (3) 8.2, (4) 6.7, (5) 7.9 , (6) 6.9 , (7) 7.3 , (8) 7.45 .

rotenone. In addition, transfer of protons into the octane occurs, as indicated by a suppression of the negative charge of the octane phase in the presence of the lipophilic proton acceptor 2,4-dinitrophenol (DNP). ${ }^{10}$ The transfer of protons ions into octane by NADH- 


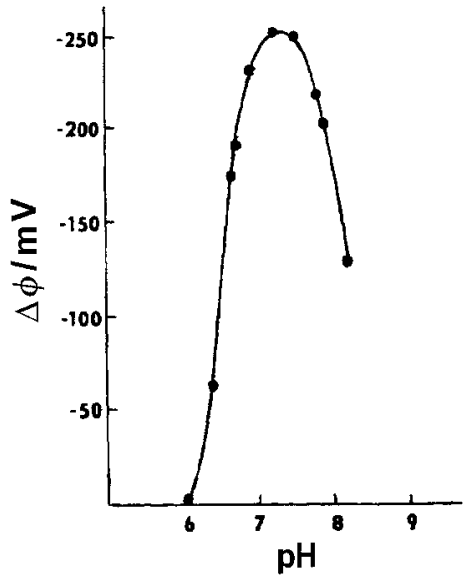

Fig. 4 Dependence of the Volta-potential at the octane/water interface on $\mathrm{pH}$. Medium: $0.02 \mathrm{M}$ Tris-HCl, $40 \mathrm{mg} / \mathrm{ml}$ cytochrome oxidase, $4 \mathrm{mM}$ ascorbate, $0.1 \mathrm{mM}$ cytochrome $c$, and $10^{-4} \mathrm{M}$ vitamin $\mathrm{K}_{3}$.

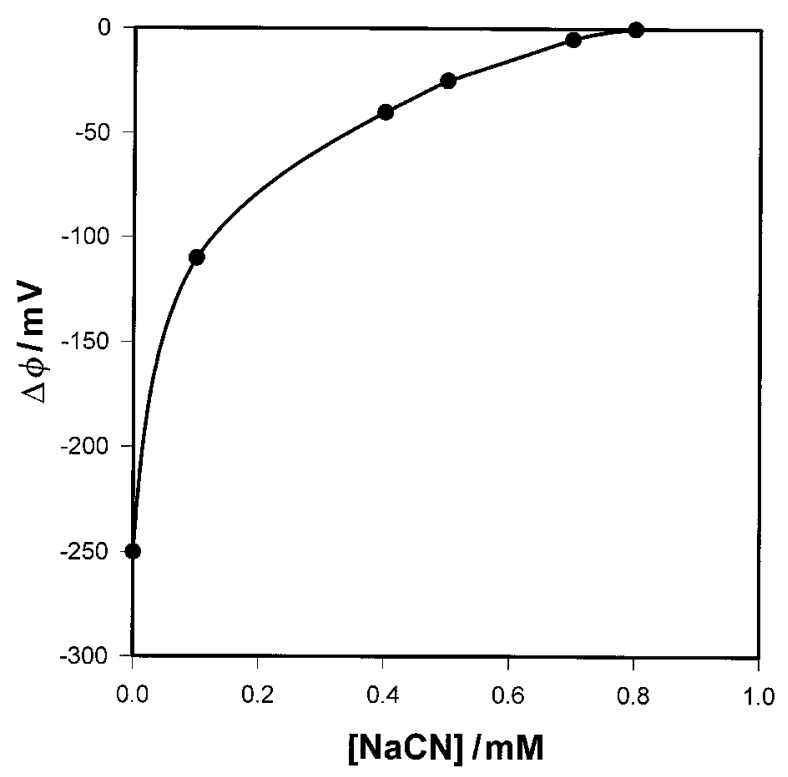

Fig. 5 Dependence of the Volta-potential at the octane/water interface on the concentration of $\mathrm{NaCN}$. Medium: $0.02 \mathrm{M}$ Tris- $\mathrm{HCl}$ ( $\mathrm{pH} 7.4), 40 \mathrm{mg} / \mathrm{ml}$ cytochrome oxidase, $4 \mathrm{mM}$ ascorbate, $0.1 \mathrm{mM}$ cytochrome $c$, and $10^{-4} \mathrm{M}$ vitamin $\mathrm{K}_{3}$.

CoQ reductase models the function of the first energy coupling site, since NADH oxidation in mitochondria generates a trans-membrane proton gradient.

The succinate-cytochrome $c$ reductase segment of the respiratory chain, which includes the second energy coupling site, contains at least two portions interacting with the nonpolar octane phase. The first probably consists of cytochrome $c$ associated with the cytochrome $c$ reductase complex and is immersed in the octane phase so that electrons can be transferred to the acceptor in the octane. In addition, catalysis by the succinate- cytochrome $c$ reductase reaction in the aqueous phase (final acceptor ferricyanide) is accompanied by the transfer of protons to a lipophilic proton acceptor in the octane phase. ${ }^{10}$ This functional response may represent the proton pump of the second energy coupling site which catalyzes the transfer of hydrogen ions through the mitochondrial membrane. It should be mentioned that the reduction of ferricyanide by this enzyme complex is insensitive to antimycin. The suppression by antimycin of the electrogenic function of the succinatecytochrome $c$ reductase complex in the octane/water system means that the transfer both of negative and of positive charges into the octane requires the transport of electrons through all the components of the complex. ${ }^{10}$

\section{Porphyrins as interfacial catalysts}

Porphyrins are known as prosthetic groups of the enzymes from the respiratory chain of mitochondria.

In the presence of the iron complex of coproporphyrin III tetramethyl ether (CP) and 2- $N$-methylamino-1,4-naphthoquinone in octane, addition of a reducing agent such as $\mathrm{NADH}$ or ascorbate into the aqueous phase leads to a shift of potential in a negative direction (Fig. 6). This change depends on the concentrations of all reaction components, and can be interpreted as a reduction of the acceptor molecules during substrate oxidation in an electron exchange reaction catalyzed by $\mathrm{CP}$.

Using the phenomenological theory of a catalytic charge-transfer process through the interface of two immiscible liquids and extrapolating the experimental curve shown in Fig. 6, the Michaelis constant can be determined. From Fig. 6 it is seen that at pH $7.3 K_{\mathrm{m}}$ is

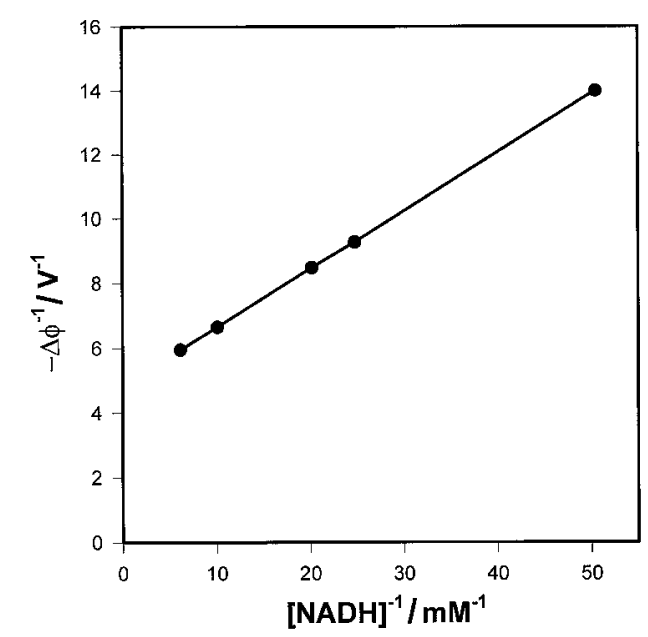

Fig. 6 Dependence of the Volta-potential on the NADH concentration in reverse coordinates. Medium: $10^{-4} \mathrm{M} 2-\mathrm{N}$ methylamino-1,4-naphthoquinone, $7 \times 10^{-2} \mathrm{M}$ Tris-HC1, $7 \times 10^{-6} \mathrm{M} \mathrm{CP}, \mathrm{pH} 7.3$. The potential is measured against an aqueous solution free of NADH. After ref. 12. 
equal to $3 \times 10^{-4} \mathrm{M}$. The interfacial potential due to the electron exchange reaction is proportional to the number of catalyst molecules located at the interface. A linear dependence indicates that the orientation of the catalyst in all substrate concentrations remains unchanged.

Electron-transfer reaction at the octane/water interface in the presence of catalytic amounts of metalloporphyrins was investigated by Volkov et al: $:^{12-14}$

$$
\begin{aligned}
& (\mathrm{NADH})_{\text {water }}+(\mathrm{MNQ})_{\text {octane }} \\
& \stackrel{\text { porphyrin }}{\rightleftharpoons}\left(\mathrm{NAD}^{+}\right)_{\text {water }}+\left(\mathrm{MNQH}^{-}\right)_{\text {octane }}
\end{aligned}
$$

This reaction occurs in the dark, and light $(70 \mathrm{~mW} /$ $\mathrm{cm}^{2}$ ) does not effect the potential change. The Voltapotential measured in chain (I) relative to the background value before the reaction started is not affected by the way in which the reaction starts, by substrate or acceptor. In the absence of one of the following reaction components, i.e. CP, octane, NADH, or naphthoquinone, no change of the Volta-potential was observed. The Volta-potential measured in chain (I) is also independent of the buffer capacity of the solvent (Tris- $\mathrm{HCl}$ concentration range from 10 to $100 \mathrm{mM}$ ). This indicates that the change in the potential jump is caused by the electron-transfer reaction. Thus, the physicochemical state of the catalyst molecules at the interface (orientation, interaction between the molecules, adsorption energy, Michaelis constant) may be studied using the adsorption isotherms and measurements of the Volta-potential in chain(I). The experimental results are in a good agreement with a previously proposed model of the catalytic charge transfer through the interface of two immiscible liquids.

\section{Reduction of porphyrin at the octane/water interface controlled by the specific adsorption: molecular recognition of anions at the octane/water interface}

The redox reaction between the ferric complex of hydrophobic porphyrin FeP (ferric complex of 2,7,12,17-tetramethy 1-3,13-octadecy 1-8,18-bis(carbomethoxyethyl) porphyrin) and sodium dithionite in two different phases occurring at the interface between two immiscible liquids has been investigated by Volta potential measurements and UV-Vis spectroscopy. ${ }^{13}$ The reduction of the ferric complex of hydrophobic porphyrin adsorbed at the interface was found to be accompanied by a potential shift in the negative direction and to depend significantly on the nature of the anion and the ionic strength of the supporting electrolyte. Specifically adsorbed halogen anions inhibited the redox reaction in the sequence: $\mathrm{Cl}^{-}<\mathrm{Br}^{-}<\mathrm{I}^{-}$. Depending on $\mathrm{pH}$, the ferric complex of hydrophobic porphyrin exists in the uncharged FeP-0-FeP form or in the cation $\mathrm{FeP}^{+}$form.

The Volta potential measurements in the chain (I) showed a positive change when plotted with respect to the water phase and increased smoothly up to $0.46 \mathrm{~V}$ with increasing FeP concentration up to $10^{-6} \mathrm{M}$. Ferric complexes of porphyrins are known to be salts in which the metal complex is a cation. When dissolved in dry hydrocarbon these salts are undissociated, but at the aqueous interface or in octane with water present as a microemulsion, the following hydrolysis reaction takes place:

$$
\mathrm{FeP}^{+} \mathrm{Cl}^{-}+\mathrm{H}_{2} \mathrm{O} \rightleftharpoons \mathrm{FeP}^{+} \mathrm{OH}^{-}+\mathrm{H}^{+}+\mathrm{Cl}^{-}
$$

The product of the reaction can undergo dimerization to form a $\mu$-complex:

$$
2 \mathrm{FeP}^{+} \mathrm{OH}^{-} \rightleftharpoons \mathrm{FeP}-\mathrm{O}-\mathrm{FeP}+\mathrm{H}_{2} \mathrm{O}
$$

Comparison of the FeP absorption spectra in dry and in wet octane showed that hydrolysis in fact transforms the $\mathrm{FeP}^{+} \mathrm{Cl}^{-}$spectrum into the $\mathrm{FeP}^{+} \mathrm{OH}^{-}$or $\mathrm{FeP}-0-\mathrm{FeP}$ spectra. ${ }^{13}$ Acidifying the aqueous solution with hydrochloric acid shifts the equilibrium of reaction (4) to the left, and the initial $\mathrm{FeP}^{+} \mathrm{Cl}^{-}$form appears again. It should be noted that addition of $1 \mathrm{M} \mathrm{KC1}$ is not sufficient for the equilibrium of the reaction (4) to shift to the left.

A second method was used to determine the form of porphyrin at the octane/water interface. It is known that the surface potential $\chi_{\mathrm{s}}$ consists of two components

$$
\chi_{\mathrm{S}}=\chi_{\mathrm{D}}+\chi_{\mathrm{G}}
$$

The first term of the sum represents the potential caused by the dipole moment of the adsorbed particles which set up a potential determined by the Helmholtz equation

$$
\chi_{\mathrm{D}}=\frac{4 \pi n_{\mathrm{S}} \mu_{\mathrm{eff}}}{\varepsilon}
$$

where $\mu_{\text {eff }}$ is the nomal component of the effective dipole moment, $n_{\mathrm{S}}$ is the surface concentration and $\varepsilon$ is the static permittivity of the medium surrounding the dipole. The second term of Eq. (6) is due to the presence of the diffuse ion layer and is determined by the well-known Gouy-Chapman equation. For charged monolayers

$$
\frac{\mathrm{d} \chi_{\mathrm{s}}}{\mathrm{d} \log c}=\frac{\mathrm{d} \chi_{\mathrm{G}}}{\mathrm{d} \log c} \approx 58 \mathrm{mV}
$$

Assuming that the area per molecule and the vertical component of the effective dipole moment remain unchanged, a study was made of the influence of the ionic strength and $\mathrm{pH}$ of the aqueous phase on the interfacial potential change. In the alkaline $\mathrm{pH}$ range the reaction is shifted toward $\mu$-complex formation, and this potential change should not depend on the supporting electrolyte concentration unless a significant reori- 
entation of adsorbed molecules takes place. Figure 7 shows the dependence of the Volta potential on $\mathrm{KCl}$ concentration at $\mathrm{pH}=8.5$ (curve 1) and $\mathrm{pH}=6.5$ (curve 2 ). The interfacial potential change remains the same when the electrolyte content increases at alkaline $\mathrm{pH}$ values. This means that the main contribution to the surface potential is made by $\chi_{D}$. In a more acid region at $\mathrm{pH}=6.5$ the electric double layer consists of FeP complexes in the octane phase and anions in water. The gradient $\mathrm{d} \chi_{\mathrm{S}} / \mathrm{d} \log c_{\mathrm{KCl}}$ is $55-60 \mathrm{mV}$ per decade of salt concentration between $10^{-4}-10^{-1} \mathrm{M} \mathrm{KCl}$. To determine whether aqueous phase anions make any contribution to the potential change, the Volta potentials of the system under study were measured in $\mathrm{KCl}, \mathrm{KBr}$ and $\mathrm{KI}$ solutions. If no specific anion adsorption at the octane/water interface takes place, $\chi_{\mathrm{s}}$ should not depend on the nature of the anion. However, as follows from the experimental values of $\chi_{\mathrm{s}}$ in the octane/water system, when $10^{-3} \mathrm{M} \mathrm{KCl}, \mathrm{KBr}$ and $\mathrm{KI}$ solutions are added to the water $\chi_{\mathrm{s}}$ measured against the gold reference electrode at $\mathrm{pH}=7$ equals 580,570 and $530 \mathrm{mV}$ respectively. The results obtained may be due to the fact that halogens are specifically adsorbed at the interface and shift $\Delta \phi$ toward more negative values in the sequence $\mathrm{Cl}^{-}, \mathrm{Br}^{-}, \mathrm{I}^{-}$.

Upon addition of sodium dithionite FeP undergoes reduction. This process may also be detected from the Volta potential shift in the negative direction. Higher sodium dithionite concentrations increase this shift, which attains a maximum value of $-110 \mathrm{mV}$. A more careful study of the FeP reduction showed the reaction to proceed only at low electrolyte concentrations. The spectrum of FeP treated with dithionite in the presence of $10^{-1} \mathrm{M} \mathrm{KC} 1$ coincides with that of the oxidized form of FeP, which indicates that no reduction takes place under these conditions.

Two explanations are possible for the observed inhi-

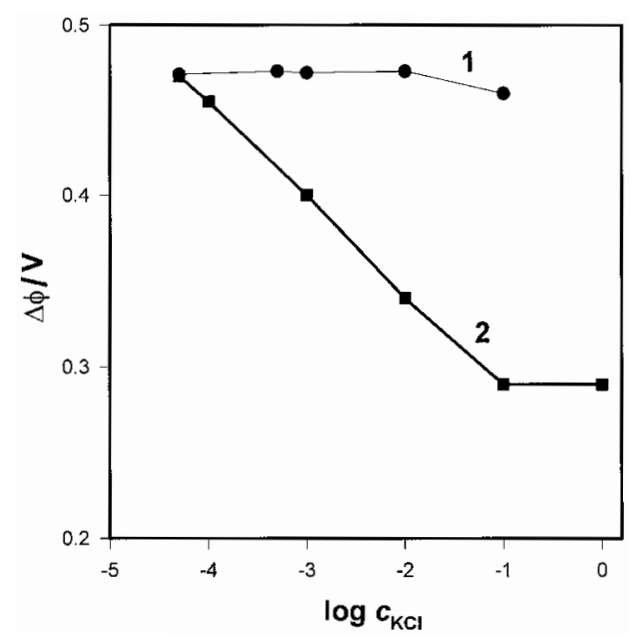

Fig. 7 Dependence of the Volta potential measured in chain (I) on $\mathrm{KCl}$ concentration in the system containing $10^{-5} \mathrm{M} \mathrm{FeP}$ at $\mathrm{pH} 8.5$ (1) and at $\mathrm{pH} 6.5$ (2). After ref. 13. bition of FeP reduction. The first assumes that the dithionite concentration is defined by the GouyChapman diffuse layer theory. As the potential at the interface decreases with increasing $\mathrm{KC} 1$ concentration, the dithionite anion concentration at the interface must also decrease, which results in a decrease in the final product concentration (reduced FeP). The second explanation assumes that dithionite anion is displaced from the interface by specifically adsorbed halides. This explanation, contrary to the first model, suggests that the observed effect of the inhibition of porphyrin reduction is based on the forces of specific adsorption. According to the first model, it does not matter what salt is used to increase the ionic strength of the solution. In the second model the concept of specific adsorption is used, and, therefore, the effect should depend on the nature of the anion. To choose between the two models, the ionic strength of the aqueous phase was increased by adding $\mathrm{Cl}^{-}, \mathrm{Br}^{-}$and $\mathrm{I}^{-}$.

Inhibition of porphyrin reduction takes place at different concentrations of halide salts, and therefore depends on the nature of the anion. ${ }^{13}$ The $\mathrm{I}^{-}$ions, which are known to have the highest adsorbability cause inhibition of the reaction at concentrations lower than that of $\mathrm{Br}^{-}$anions, which, in their turn, are more effective than $\mathrm{Cl}^{-}$anions. For the dithionite anion to transfer an electron to adsorbed porphyrin, it must be near the porphyrin molecule and occupy a position in the aqueous phase near the interface. The inhibition of porphyrin reduction as halide salt concentration increases means that halides displace dithionite anions from the adsorption layer.

\section{Conclusion}

Hydrophobic porphyrin adsorbed at the octane/water interface can be reduced with a soluble agent exclusively in the aqueous phase. This process can be recorded by the measurement of the Volta potential change in the chain (I). The influence of specifically adsorbed anions on the reduction process suggests that the GouyChapman model may not provide a complete description of the electric double layer at the interface between two immiscible liquids or at the membrane/electrolyte interface.

Processes occurring at the interface between two immiscible liquids present a challenge of considerable interest because heterogeneous structures of this kind are frequently encounted in nature. Future research in this area will provide further insight into both biological and chemical application of molecular recognition and interfacial catalysis at liquid/liquid interfaces.

\section{References}

1. A. G. Volkov and D. W. Deamer (ed.), "Liquid-Liquid Interfaces: Theory and Methods", CRC Press, Boca Raton, 
London, Tokyo, 1996.

2. A. G. Volkov, M. I. Gugeshashvili and D. W. Deamer, Electrochim. Acta, 40, 2849 (1995).

3. A. G. Volkov and D. W. Deamer, Progr. Colloid Polym. Sci., 103, 21 (1997).

4. A. G. Volkov, D. W. Deamer, D. L. Tanelian and V. S. Markin, Progr. Surf. Sci., 53, 1 (1996).

5. A. G. Volkov, D. W. Deamer, D. L. Tanelian and V. S. Markin, "Liquid Interfaces in Chemistry and Biology", Wiley, New York, 1997.

6. S. Kihara, M. Suzuki, K. Maeda, K. Ogura, M. Matsui and Z. Yoshida, J. Electroanal. Chem., 271, 107 (1989).

7. Yu. I. Kharkats and A. G. Volkov, Biochim. Biophys. Acta, 891, 56 (1987).

8. Yu. I. Kharkats and A. G. Volkov, J. Electroanal. Chem., 184, 435 (1985).

9. L. I. Boguslavsky, A. G. Volkov, A. A. Kondrashin, S. T.
Metelsky and A. A. Yasaitis, Biokhimiya, 41, 1047 (1976).

10. L. I. Boguslavsky, A. G. Volkov, A. A. Kondrashin, V. P. Skulachev and A. A. Yasaitis, Bioorg. Khim., 1, 1783 (1975).

11. L. I. Boguslavsky, A. A. Kondrashin, I. A. Kozlov, S. T. Metelsky, V. P. Skulachev and A. G. Volkov, FEBS Lett., 50, 223 (1975)

12. A. G. Volkov, M. A. Bibikova, A. F. Mironov and L. I. Boguslavsky, Bioelectrochem. Bioenerg., 10, 477 (1983).

13. A. G. Volkov, M. I. Gugeshashvili, A. F. Mironov and L. I. Boguslavsky, Bioelectrochem. Bioenerg., 9, 551 (1982).

14. A. G. Volkov, M. I. Gugeshashvili, A. F. Mironov and L. I. Boguslavsky, Bioelectrochem. Bioenerg., 10, 485 (1983).

(Received October 8, 1997) (Accepted November 26, 1997) 